\title{
Investigative Interviews with Youth Victims of Human Trafficking for Sexual Purposes in Sweden
}

\author{
Johanna Lindholm*, Ann-Christin Cederborg* \& Charlotte Alm** \\ *Department of Child and Youth Studies \\ Stockholm University \\ S-106 91 Stockholm \\ Sweden \\ **Department of Psychology \\ Stockholm University \\ S-106 91 Stockholm \\ Sweden
}

\begin{abstract}
This study examines the quality of questions asked by police officers and responses elicited from youths subjected to human trafficking for sexual purposes in Sweden. Preliminary results show that the police officers ask few invitations but a large number of option-posing questions. These latter questions elicited information but the responses may not be extensive or accurate. The police officers' use of directive questions and social pressure was associated with evasive responses. This finding seems to be due to the content of these utterances as they concern the perpetrators' activities or details about the girls' actions.
\end{abstract}

\section{Summary}

Previous studies on human trafficking concern, for example, treatment and protection of exposed children and identification of child victims (Bokhari, 2008; Gozdziak, 2010). So far studies on how police officers investigate child trafficking is limited.

Many studies on investigative interviewing have shown that children can provide accurate reports from 4 years of age, but they might be influenced by suggestive questions. Hence, elicited details and level of accuracy are heavily reliant on the ways in which children are interviewed (Malloy et al., 2011). Invitations elicit information that is 
much more likely to be accurate and three times more informative than information elicited using option-posing and suggestive prompts (Lamb et al., 1996).

This study explores how investigative interviews with victims of human trafficking are performed. It is part of a larger project about human trafficking in Sweden. Our data consist of court files and preliminary investigations of all cases treated by the Swedish court since the law against human trafficking was introduced in 2002.

\section{Method}

We examine the first transcribed interview with 22 girls. They were between 14,8 and 18,1 years of age $(M=16,9)$ when interviewed.

The first author assessed each question using categories developed for forensic research interviews (Lamb et al., 1996). The second author independently coded $20 \%$ of the transcript. There was $96 \%$ inter-rater reliability. We distinguished between the following question types:

Invitations: utterances prompting free-recall responses from the child.

Directives: refocus the child's attention on details or aspects that she had already mentioned, requesting additional information.

Option-posing: focus the child's attention on details or aspects that the child had not previously mentioned, asking her to affirm, negate or select interviewer given options. Suggestive: utterances stated in such a way that the interviewer strongly communicate what responses are expected or assume details that have not been disclosed by the child.

Summaries: utterances summarizing the information previously provided by the child. Social support: involves interviewer's support of the child's behaviour and attempts to normalize the situation.

Social pressure: involves criticism of the child's behaviour and actions. 
The children's responses to the rendered questions were then coded using categories inspired by Keselman et al., (2010). The first author categorized each of the minors' responses. The second author independently coded $20 \%$ of the transcripts. There was $95 \%$ inter-rater reliability. We distinguished between the following categories:

\section{Disclosures}

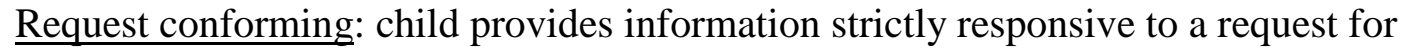
information.

Agreements: child agrees verbally with the interviewer's proposed option/restatement. Disagreements: child disagrees verbally with, and sometimes corrects, the interviewer's proposed option/restatement.

Extended task-related: additional task-related information volunteered by the child beyond the scope of information requested.

\section{Non-disclosures}

No answer: no request or task-related information.

Evasive response: unclear references, imprecise feedback and vague descriptions.

In the third stage of the analysis, we qualitatively assessed the content of question type in relation to type of response given.

\section{Results}

So far, we have coded all questions posed by the police officers in 22 interviews and all responses given by the girls in 10 interviews. We have also qualitatively assessed the content of question types in relation to type of responses given in these 10 cases. The current analysis involves interviews with 10 girls comparing question types and responses.

In total, these children were asked 3381 questions. A one way ANOVA showed that option-posing questions $(41,3 \%)$ were the most used prompt followed by directives $(29,3$ 
$\%)$ and summaries $(11,5 \%)$. Few suggestive questions $(5,6 \%)$ and invitations $(2,2 \%)$ were asked.

A one way ANOVA showed that request confirmations (40.2\%) were the most used responses. Pearson correlations computed between the different question types and responses showed a correlation between directive questions and evasive responses $(r=.92, p$ $<.001)$, as well as social pressure and evasive responses $(r=.82, p<.01)$. Option- posing questions correlated with request confirming $(r=.93, p<.001)$, agreements $(r=.94, p<$ $.001)$ and disagreements $(r=.95, p<.001)$.

The preliminary qualitative analysis showed that evasive responses were elicited from questions about the perpetrators' activities or details about the girls' actions.

\section{Discussion}

The findings shed light on what prerequisites children subjected to human trafficking are given when interviewed about their experiences. It also gives an insight in the girls' willingness to report about their experiences. So far the findings indicate that the girls are not given satisfying opportunities to report because of the extensive use of option-posing questions. Although such questions elicit information, the responses may not be as extensive or accurate as if they were asked invitations (Lamb et al., 1996). Another finding is that the police officers' use of directives and social pressure may result in evasive responses. This latter finding seems to be due to the content of these utterances as they concern the perpetrators' activities or details about the girls' actions. Further analysis will deepen our understanding of why the girls to a limited extent expand their responses. 


\section{References}

Bokhari, F. (2008). Falling through the gaps: safeguarding children trafficked into the UK. Children \& Society, 22, 201-211.

Gozdziak, E. M. (2010). Identifying child victims of trafficking. Criminology \& Public Policy, 9, (2), 245-255.

Keselman, O., Cederborg, A-C., Lamb, M. E., \& Dahlström, Ö. (2010). Asylum-seeking minors in interpreter-mediated interviews: what do they say and what happens to their responses? Child \& Family Social Work, 15, (3), 325-334.

Lamb, M. E., Hershkowitz, I., \& Sternberg, K. J. (1996). Effects of investigative utterance types on Israeli children's responses. International Journal of Behavioral Development, 19, (3), 627-637.

Malloy, L. C., La Rooy, D. J., Lamb, M. E., \& Katz, C. (2011). Developmentally sensitive interviewing for legal purposes. In M. E. Lamb, D. J. La Rooy, L. C. Malloy, \& C. Katz (Eds.), Children's Testimony: A Handbook of Psychological Research and Forensic Practice (1-14) Wiley-Blackwell. 